\title{
CWO4.pdf
}

\section{Materials Processing with Femtosecond Vortex Pulses}

\author{
Cyril Hnatovsky ${ }^{1}$, Vladlen G. Shvedov ${ }^{1,2}$, Wieslaw Krolikowski ${ }^{1}$, Andrei V. Rode $^{1}$ \\ ${ }^{1}$ Laser Physics Centre and ${ }^{2}$ Nonlinear Physics Centre, Research School of Physics and Engineering, \\ The Australian National University, Canberra ACT 0200, Australia; \\ e-mailchn111@physics.anu.edu.au
}

\begin{abstract}
We present the first results on material processing with tightly focused single femtosecond laser vortex pulses. We use double-charge femtosecond vortices to produce micron-size ring-shaped structures with $<100 \mathrm{~nm}$ uniform groove thickness.

OCIS codes: (350.3390) Laser materials processing; (050.4865) Optical vortices; (320.7080) Ultrafast devices
\end{abstract}

High intensity ultrashort (femtosecond) optical vortex pulses provide a unique opportunity to investigate the effects of the optical angular momentum on atomic or molecular systems and transient non-equilibrium states of matter. Unfortunately, studies involving interactions of such pulses with matter pose significant challenges due to technical difficulties associated with their generation as the current methods using spiral phase plates and holograms are inherently chromatic and require correcting elements to compensate topological charge dispersion caused by the broad spectral bandwidth associated with ultrashort light pulses.

In this work we synthesize double-charge femtosecond vortex pulses using a polarization singularity beam converter based on light propagation in a uniaxial anisotropic medium and then tightly focus the pulses on the surface of glass samples to produce sub-micron ring structures [1,2]. Our beam converter has the following advantages:

i) suitability for the operation with high energy pulses;

ii) high conversion efficiencies of $50 \%$ for double-charge vortex beams;

iii) no topological charge dispersion for double-charge vortices, and

iv) adaptability with minimum adjustment for a wide range of wavelength and beam power conditions. Figure 1(a) shows the experimental setup for the generation of double-charge femtosecond vortex beams [2].

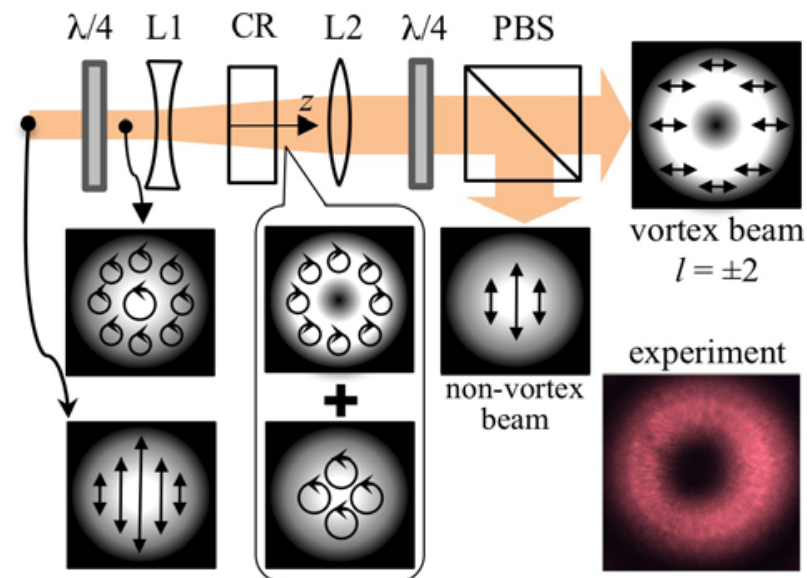

Fig. 1. Schematic representation for a beam converter for the generation of double-charge femtosecond vortex pulses. $\lambda / 4$ - achromatic quarterwave plate, L1 - negative lens, CR - uniaxial crystal, L2 - positive lens, PBS - polarization beamsplitter. On the right: Polarization states after each optical element are indicated by arrows (top); and CCD image $\left(4.2 \times 4.2 \mathrm{~mm}^{2}\right)$ of a double-charge vortex recorded $\sim 5$ meters away from the converter (bottom).

In our experiments we used the output beam of a MXR-Clark Ti:Sapphire amplifier with a central wavelength at $775 \mathrm{~nm}$. The converter transformed this beam into a linearly polarized double-charged vortex beam $(l= \pm 2, l$ is the topological charge of a beam) with the radial intensity distribution well approximated by an expression describing Laguerre-Gaussian beams [2]:

$$
I=\frac{2^{|l|+1} r^{2|l|}}{|l| \pi} \frac{\exp \left(-2 r^{2} /\left(w_{0} \mid \xi\right)^{2}\right)}{\left(w_{0} \mid \xi\right)^{2(l \mid+1)}} P,
$$




\section{CWO4.pdf}

where $P$ is the total power, $\xi=1+i z \lambda /\left(\pi w_{0}^{2}\right)$ and $w_{0}$ is the beam waist. For a vortex with $l= \pm 2$ the peak intensity is by a factor of $2 \mathrm{e}^{-2}$ lower than for a Gaussian beam (i.e., $l=0$ ) with the same $1 / \mathrm{e}^{2}$ radius. The beam was focused on to the sample surface using a NA $=0.9$ (Nikon M Plan 100x) microscope objective. The peak intensity at the focus was $\sim 10^{14} \mathrm{~W} / \mathrm{cm}^{2}$, which exceeds by far the ionization threshold of glass $\sim 10^{13} \mathrm{~W} / \mathrm{cm}^{2}$.

By controlling the light intensity we consistently machine sub-micron ring-shaped structures with less than 100 $\mathrm{nm}$ uniform groove thickness using a single laser pulse. Scanning electron microscope (SEM) images in Fig. 2(a) show the ablation rings produced at different pulse energies. In the leftmost image in Fig.2a the diameter of the ablation ring is $\sim 1 \mu \mathrm{m}$ and the width of the ablated annular groove is less than $100 \mathrm{~nm}$. According to measurements performed with a Veeco MultiMode ${ }^{\circledR} 8$ scanning probe microscope, the grooves are $\sim 90 \mathrm{~nm}$ deep, thus exhibiting a $\sim 1: 1$ depth-to-height aspect ratio. At higher pulse energies the ablation signature is much more pronounced, but it still preserves an annular shape with sharp and clean edges. The ablation of material performed using a single vortex pulse is highly reproducible, as shown in Fig. 2(c).
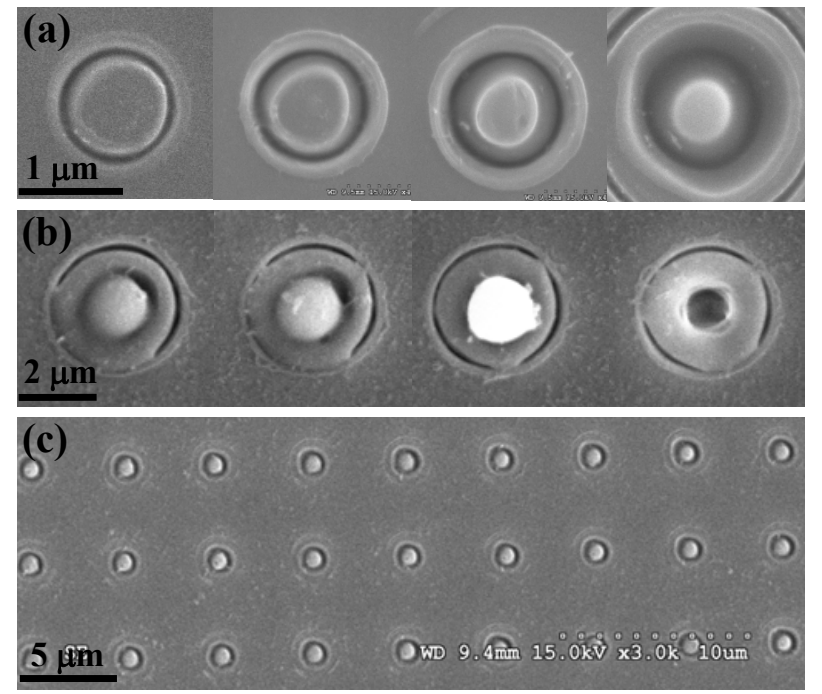

Fig. 3. Ablation of soda-lime glass with 200 fs double-charge single femtosecond vortex pulses using NA $=0.9$ focusing optic. (a) ablation rings produced at a pulse energy of $\sim 100 \mathrm{~nJ}, \sim 150 \mathrm{~nJ}, \sim 250 \mathrm{~nJ}$, and $\sim 700 \mathrm{~nJ}$ from left to right. (b) cavities produced at a pulse energy of $\sim 1.5 \mu \mathrm{J}$. (c) a region of a $200 \times 200 \mu \mathrm{m}^{2}$ array produced at a pulse energy of $\sim 250 \mathrm{~nJ}$. Ablation rings are separated by $5 \mu \mathrm{m}$.

An interesting effect is observed when the pulse energy is increased to a maximum of $\sim 1.5 \mu \mathrm{J}$ and the laser focus is positioned $\sim 2 \mu \mathrm{m}$ subsurface (Fig. 2(b)). Now we clearly observe the formation of deep cavities on the axis of the focused beam where the residual central light intensity is negligible. The $\sim 1.5 \mu \mathrm{m}$ deep cavity in the end of the row has opened, whereas the cavities to the left of it are still covered with material to a different extent. We suggest that the formation of nanocavities with a single femtosecond vortex pulse is caused by the implosion phenomenon. By focusing a femtosecond vortex pulse very tightly, we concentrate the electromagnetic energy inside a micrometersize toroidal volume where the material is converted into toroidally shaped hot electron plasma. The plasma generates a converging cylindrical shock wave which can reach the axis of the toroid and raise pressure inside that region dramatically. The implosion phase is followed by the explosion of the ultra-compressed material near the surface, which leads to the formation of cavities.

\section{References:}

[1] V. G. Shvedov, C. Hnatovsky, W. Krolikowski, A. V. Rode. "Efficient beam converter for the generation of high power femtosecond vortices." Opt. Lett. 35, 2660-2662 (2010).

[2] C. Hnatovsky, V. G. Shvedov, W. Krolikowski, A. V. Rode. "Materials processing with a tightly focused femtosecond laser vortex pulse." Opt. Lett. 35, 3417-3419 (2010). 